\title{
Caesarean delivery on maternal request (CDMR): clinical and ethical dilemma
}

Sir,

This is in reference to the research article published in your journal in feburary 2017 titled "Is current rising trend of cesarean sections justified?" by Preetkamal, Harmanpreet Kaur, Madhu Nagpal.

Now a day, in developing countries like India, one of the most common contributing factor for increasing caesarean section rate is CDMR (Caesarean delivery on maternal request). Caesarean delivery on maternal request (CDMR) is defined as a primary caesarean delivery performed at the request of mother in the absence of any medical or obstetric indication. Incidence of CDMR reached up to 18 percent of total caesarean deliveries worldwide. ${ }^{1}$ The increasing prevalence of CDMR may be accounted for the perceived advantages of procedure for mothers, babies and healthcare providers.

However, The International Federation of Gynaecology and Obstetrics states that performing caesarean section in absence of medical indication is unethical whereas Society of Obstetricians and Gynaecologists of Canada states that caesarean section should be reserved for those pregnancies in which there is a threat to the health of the mother and/or the baby but a mother may request a caesarean section with the belief that there is real benefit to herself and her baby, or as result of anxiety over labour, the birthing process and its potential consequences. $^{2}$

A planned caesarean section is also viewed by mother as a safer mode of delivery because it may reduce the risk of intrapartum death, hypoxia and birth trauma. Caesarean section also eliminates detrimental intrapartum events such as shoulder dystocia or non-progress of labour and reduce the risk of meconium aspiration and lowers possibility of intubation in infants. Conversely, a planned vaginal delivery places neonates at an increased risk of intracranial haemorrhage, asphyxia, encephalopathy and infection. Yokoe et al showed that emergency caesarean deliveries were associated with greater incidence of postpartum infection than elective procedures. $^{3}$ Most women desire caesarean births to avoid the potential for perineal trauma associated with normal and assisted vaginal delivery.

We are at turning point in obstetric thinking, brought about not only by the advances that have made caesarean section safe and evidence that vaginal delivery can be associated with substantial morbidity but also by attitude of our society, which reflect intolerance to risk. We encourage" family planning", pre-pregnancy counselling, we routinely perform antenatal screening and we offer prenatal diagnosis- all promote a concept of "designer baby". Can we do all this and refuse women a safe mode of delivery (caesarean section) that removes gambles associated with labour and which she personally finds unacceptable?

The obstetrician is guided by four principles of medical ethics: autonomy, justice, non-maleficence and beneficence and patient may view their request for delivery by caesarean section as exercising their right to individual choice and self-governance.

The current approach should be to discuss the risks and benefits of caesarean section with mother. Patient's requests should be non-directive counseling, which incorporates the women's values and cultural context with sensitivity to her concerns. ${ }^{4}$ The dialogue should aim to maximize the patient's understanding of issues and focus on individual needs, including future reproductive plans, medical risk factors, and psychological concerns. After a thorough discussion and review, caesarean delivery on maternal request may be agreed upon as reasonable alternative to planned vaginal delivery.

\section{CONCLUSION}

The issue of CDMR is ongoing bioethical debate which highlights the struggle to balance patient autonomy with duty of obstetrician to uphold the principle of beneficence, non-maleficence and justice. Our mission should be to promote safe, effective and satisfying maternity care for all women and their families through research, education, expertise and advocacy.

"It doesn't matter when, where or how you gave birth. What matters is how you cherish every moment after".

\section{Ankita Mann*, Sumita Mehta, Anshul Grover}

Department of Obstetrics and Gynecology, BJRM Hospital, New Delhi, India

*Correspondence:

Dr. Ankita Mann,

E-mail: ankitamann1988@gmail.com 


\section{REFERENCES}

1. Kalish RB, McCullough LB, Chervenak FA. Patient choice caesarean delivery: ethical issues. Curr Opin Obstet Gynecol. 2008;20(2):116-9.

2. Society of Obstetricians and Gynaecologists of Canada, the Association of Women's Health, Obstetric and Neonatal Nurses of Canada, the Canadian Association of Midwives, the College of Family Physicians of Canada, the Society of Rural Physicians of Canada. Joint policy statement on normal childbirth. J Obstet Gynecol Can 2008;30:1163-5.

3. Yokoe DS, Christiansen CL, Johnson R, Sands K, Livingston J, Shtatland ES et al. Epidemiology of and Surveillance for Postpartum Infections. Emerg Infect Dis. 2001;7(5):837-841.

4. American College of Obstetricians and Gynecologists. ACOG Committee Opinion. Caesarean delivery on maternal request. Obstet Gynecol. 2007;110(5):1209-12.

Cite this article as: Mann A, Mehta S, Grover A. Caesarean delivery on maternal request (CDMR): clinical and ethical dilemma. Int $\mathbf{J}$ Reprod Contracept Obstet Gynecol 2017;6:3201-2. 